\section{Perfil dos usuários de crack que buscam atendimento em Centros de Atenção Psicossocial}

\author{
Crack cocaine users who attend outpatient services
}

\begin{abstract}
This paper describes the profile of 95 crack cocaine users attending three community mental health services (CAPS) in Greater Metropolitan Porto Alegre, Rio Grande do Sul State, Brazil, from August 2009 to March 2010. The instruments employed were questionnaires developed by the team, the Self-Reporting Questionnaire (SRQ-20), and inventories of criteria for dependence and abuse (SAMHSA). The data depict a group of users consisting predominantly of young males with elementary schooling, without regular employment but reporting individual income, none of whom living on the streets. They were currently addicted, with heavy daily use of crack for more than two years, and with high SRQ-20 score. This group's characteristics showed that the community mental health services are attended by crack users that suffer losses resulting from their addiction, but also some possible selection process in the supply of these health services (based mainly income, schooling, or primary support network).
\end{abstract}

Crack Cocaine; Drug Users; Mental Health Services
Rogério Lessa Horta 1,2

Bernardo Lessa Horta 3

Adriana Palma Rosset 1

Cristina Lessa Horta 2

\section{Introdução}

O consumo de substâncias tem elevada prevalência no Brasil 1 , gerando diferentes demandas em atenção à saúde. O crack, cocaína fumada, tem elevado potencial de gerar danos diversos $2,3,4,5,6,7$. Sintomas depressivos e ansiosos, sejam comorbidades ou decorrência do consumo ${ }^{8}$, determinam menos motivação para a mudança e menor adesão ao tratamento 9,10,11.

$\mathrm{O}$ atendimento aos usuários de drogas no Sistema Único de Saúde (SUS) tem os Centros de Atenção Psicossocial (CAPS) 12 como referência. Os CAPS garantem a oferta de atendimento especializado próximo ao local de moradia dos usuários, mas têm sido descritos problemas no acesso, além de evidências de estigmatização no vínculo com os CAPS 13.

O estudo do crack é recente no Brasil 4 . Maior número de usuários, maior visibilidade social e maior demanda sobre os serviços de saúde levam à necessidade de ampliação deste campo de estudo.

O planejamento de ações nessa área depende de maior apropriação de dados sobre a população usuária do crack e daqueles que buscam atendimento no sistema público de saúde. Estudos de resolutividade dos serviços precisam ser precedidos pelo reconhecimento do perfil da população que chegará aos serviços. Eventuais seletividades põem em risco o princípio da universalidade que rege o SUS. 
É interesse deste estudo conhecer o perfil de usuários de crack acolhidos em CAPS de três municípios da Região Metropolitana de Porto Alegre, no Rio Grande do Sul, Brasil.

\section{Método}

Estudo exploratório descritivo que entrevistou, entre agosto de 2009 e março de 2010, usuários de crack que buscaram atendimento nos CAPS dos municípios de Igrejinha e Taquara (CAPS I), e Novo Hamburgo (CAPS AD). Os municípios foram escolhidos por conveniência, por terem diferentes portes e distintos estágios de organização das redes locais de saúde. Cada indivíduo foi convidado a passar por uma entrevista composta por:

- Questionário padronizado, desenvolvido pela equipe, com dados sociodemográficos, configuração do grupo familiar e da moradia, e histórico e padrão de consumo do crack;

- Inventários para diagnósticos de dependência e de abuso para drogas ilícitas 14 da Substance Abuse and Mental Health Services Administration (SAMHSA), em versão traduzida para o português, não validada para a população brasileira, com questões de respostas "sim" / "não". São considerados dependentes os indivíduos com 3 respostas "sim" das 7 condições propostas; e para abuso, 1 "sim" entre as 4 condições indicadas;

- Self-Reporting Questionnaire (SRQ-20), instrumento de screening de distúrbios psiquiátricos menores (DPM), recomendado pela Organização Mundial da Saúde (OMS) e validado para a população brasileira 15. Das 20 questões com respostas "sim” / "não", é considerado positivo para DPM a soma de 6 ou mais respostas afirmativas para homens e 8 ou mais para mulheres.

Chegaram aos CAPS 101 indivíduos. Seis entrevistas foram excluídas e estes usuários não retornaram mais ao serviço e não foram localizados em três tentativas de busca ativa. A relação de entrevistados e os principais dados de identificação eram sistematicamente checados com os dados de prontuário e relatórios dos CAPS para controle de qualidade e prevenção de perda amostral. Os dados foram digitados em dupla entrada, para controle de inconsistências, no programa SPSS 17.0 (SPSS Inc., Chicago, Estados Unidos). O artigo apresenta os resultados da análise descritiva dos dados obtidos.

Este estudo foi aprovado pelo Comitê de Ética em Pesquisa da Universidade do Vale do Rio dos Sinos (UNISINOS; Resolução no. 023/2009) e teve apoio financeiro do Conselho Nacional de Desenvolvimento Científico e Tecnológico (CNPq; edital 033/2008 - Saúde Mental).

\section{Resultados}

Predominaram nos CAPS usuários masculinos de crack, adultos jovens, com escolaridade fundamental ou média, sem ocupação regular, mas com renda individual informada. As variáveis sociodemográficas e os dados que descrevem seus grupos familiares e a composição dos domicílios são apresentadas na Tabela 1. Prevaleceram indivíduos solteiros, que não coabitavam com companheiro/a, vivendo em domicílios com 2 a 4 pessoas. Apenas 5,3\% viviam sós e nenhum se disse em condição de rua. Uma parte desses indivíduos (30,5\%) não informou renda familiar, mas nenhum declarou não possuir renda. Os usuários informaram ter filhos, mas a maioria $(72,5 \%$ dos que tinham filhos) referiu não coabitar com eles.

A Tabela 2 mostra o padrão de consumo e problemas associados, com evidência de uso por mais de um ano antes de chegar aos CAPS, com idades de início do consumo acima de 18 anos de idade, com o uso em companhia de outras pessoas, diário e em grandes quantidades. O número de pedras referido pelos usuários, por episódio típico de consumo, foi igual ou superior a $10 \mathrm{em}$ 69,5\% dos casos. Nicotina, álcool e maconha foram, respectivamente, as drogas mais referidas como substâncias usadas antes do crack e também em associação com este. Maconha é, entre as três, a substância com o menor percentual de usuários desejando cessar o consumo (24\%).

Ainda na Tabela 2, aparece a ocorrência de estressores psicossociais nos 12 meses anteriores às entrevistas, destacando-se o envolvimento referido com polícia ou justiça, que quase todos mencionaram. Do ponto de vista clínico, grande parte dos entrevistados preenchia critérios para dependência, abuso e DPM.

\section{Discussão}

Os poucos artigos publicados sobre este tema têm base comunitária ou em serviços, mas não em CAPS, como este. Um estudo de revisão 16 apontou que a quase totalidade de usuários de crack descritos na literatura eram homens, muito jovens, pobres, analfabetos e de famílias desestruturadas. Já nos artigos que descrevem os usuários em atendimento 6,17,18,19,20, o perfil é mais próximo ao descrito aqui.

A distribuição dos usuários segundo o sexo, semelhante em todos os estudos, remete à determinação histórica e social do fenômeno, envolvendo questões de gênero 21,22. Em 2005, no Brasil, homens consumiam mais crack do que mulheres. No Sul, a diferença reduzia, com o 
Perfil sociodemográfico de usuários de crack que buscaram atendimento em Centros de Atenção Psicossocial (CAPS) em 2009/2010 no Sul do Brasil ( $n=95)$.

\begin{tabular}{|c|c|c|}
\hline \multirow[t]{2}{*}{ Variáveis/Categorias } & \multicolumn{2}{|c|}{ Distribuição } \\
\hline & $\%$ & $\mathbf{n}$ \\
\hline \multicolumn{3}{|l|}{ Sexo } \\
\hline Masculino & 82,1 & 78 \\
\hline Feminino & 17,9 & 17 \\
\hline \multicolumn{3}{|l|}{ Idade (anos) } \\
\hline Até 20 & 18,9 & 18 \\
\hline $21-30$ & 51,6 & 49 \\
\hline Acima de 30 & 29,5 & 28 \\
\hline \multicolumn{3}{|l|}{ Escolaridade em anos completos } \\
\hline Até 8 & 56,8 & 54 \\
\hline 9 ou mais & 43,1 & 41 \\
\hline \multicolumn{3}{|l|}{ Ocupação regular } \\
\hline Sim & 27,4 & 26 \\
\hline Não & 72,6 & 69 \\
\hline \multicolumn{3}{|c|}{ Informou renda na entrevista inicial } \\
\hline $\operatorname{Sim}$ & 61,0 & 58 \\
\hline Não & 39,0 & 37 \\
\hline \multicolumn{3}{|l|}{ Estado civil } \\
\hline Solteiros & 64,2 & 61 \\
\hline Separados ou divorciados & 12,6 & 12 \\
\hline Casados ou união estável & 23,1 & 22 \\
\hline \multicolumn{3}{|l|}{ Coabita com companheira/o } \\
\hline $\operatorname{Sim}$ & 26,3 & 25 \\
\hline Não & 73,7 & 70 \\
\hline \multicolumn{3}{|c|}{ Número de moradores no domicílio (inclui o usuário) } \\
\hline Vivendo só & 5,3 & 5 \\
\hline 2 a 4 pessoas & 66,3 & 63 \\
\hline 5 ou mais pessoas & 28,4 & 27 \\
\hline \multicolumn{3}{|c|}{ Renda familiar (salários mínimos) } \\
\hline Não informada & 30,5 & 29 \\
\hline Até 2 & 36,8 & 35 \\
\hline Acima de 2 & 32,6 & 31 \\
\hline \multicolumn{3}{|l|}{ Numero de filhos/as } \\
\hline Nenhum & 46,3 & 44 \\
\hline 1 & 25,2 & 24 \\
\hline 2 & 28,4 & 27 \\
\hline \multicolumn{3}{|c|}{ Coabitavam com filhos/as (para quem informou ter filhos/as) } \\
\hline $\operatorname{Sim}$ & 27,5 & 14 \\
\hline Não & 72,5 & 37 \\
\hline
\end{tabular}

risco relativo (RR) para o consumo de crack entre os homens igual a 5,5, tendo o consumo pelas mulheres como referência, enquanto no Brasil todo o RR era de 7,5:1 1. Nos CAPS deste estudo, a proporção de homens que buscaram atendimento em função do crack, em relação às mulheres, caiu para 4,5:1. Maior participação de mulheres na clientela dos CAPS pode apenas acompanhar a tendência delas buscarem mais os serviços de saúde que os homens 22,23, mas garantia de vagas para as usuárias de crack, principalmente em período gestacional, deve ser lembrada 24,25 . 
Características de consumo e problemas associados entre usuários de crack que buscaram atendimento em Centros de Atenção Psicossocial (CAPS) em 2009/2010 no Sul do Brasil $(n=95)$.

\begin{tabular}{|c|c|c|}
\hline \multirow[t]{2}{*}{ Variáveis/Categorias } & \multicolumn{2}{|c|}{ Distribuição } \\
\hline & $\%$ & $\mathbf{n}$ \\
\hline \multicolumn{3}{|l|}{ Tempo de uso do crack (anos) } \\
\hline Até 1 & 15,8 & 15 \\
\hline $1-2$ & 33,7 & 32 \\
\hline Mais de 2 & 50,5 & 48 \\
\hline \multicolumn{3}{|l|}{ Idade de início do uso do crack } \\
\hline Até 18 anos & 30,5 & 29 \\
\hline Acima de 18 anos & 69,5 & 66 \\
\hline \multicolumn{3}{|l|}{ Frequência de consumo } \\
\hline Diário & 50,5 & 48 \\
\hline Até 6 vezes por semana & 49,5 & 47 \\
\hline \multicolumn{3}{|c|}{ Número de pedras por episódio típico de consumo } \\
\hline Até 9 & 30,5 & 29 \\
\hline 10 ou mais & 69,5 & 66 \\
\hline \multicolumn{3}{|l|}{ Com quem costuma usar } \\
\hline Com outras pessoas & 54,7 & 52 \\
\hline Sozinho & 45,3 & 43 \\
\hline \multicolumn{3}{|l|}{ Coabitam com usuários } \\
\hline De crack & 16,8 & 16 \\
\hline De outras drogas & 52,6 & 50 \\
\hline De crack ou outras drogas & 53,7 & 51 \\
\hline \multicolumn{3}{|c|}{ Usava outras substâncias antes do crack } \\
\hline Sim & 78,9 & 75 \\
\hline Não & 21,1 & 20 \\
\hline \multicolumn{3}{|c|}{ Quais usava antes (apenas para quem informou usar) } \\
\hline Nicotina & 62,7 & 47 \\
\hline Álcool & 60,0 & 45 \\
\hline Maconha & 58,7 & 44 \\
\hline \multicolumn{3}{|c|}{ Usa outras substâncias além do crack } \\
\hline Sim & 82,1 & 78 \\
\hline Não & 17,9 & 17 \\
\hline \multicolumn{3}{|c|}{ Quais usa além (apenas para quem informou usar) } \\
\hline Nicotina & 65,4 & 51 \\
\hline Álcool & 56,4 & 44 \\
\hline Maconha & 48,7 & 38 \\
\hline \multicolumn{3}{|c|}{ Deseja cessar uso de outras substâncias (apenas para quem } \\
\hline \multicolumn{3}{|l|}{ informou usar) } \\
\hline $\operatorname{Sim}$ & 76,9 & 60 \\
\hline Não & 23,1 & 18 \\
\hline \multicolumn{3}{|c|}{ Quais deseja cessar (apenas para quem informou usar) } \\
\hline Nicotina & 43,6 & 34 \\
\hline Álcool & 37,2 & 29 \\
\hline Maconha & 24,3 & 19 \\
\hline
\end{tabular}

(continua) 


\begin{tabular}{|c|c|c|}
\hline \multirow[t]{2}{*}{ Variáveis/Categorias } & \multicolumn{2}{|c|}{ Distribuição } \\
\hline & $\%$ & n \\
\hline \multicolumn{3}{|c|}{ Escores no SRQ-20 (critérios para distúrbios psiquiátricos menores) } \\
\hline Positivo & 78,9 & 75 \\
\hline Negativo & 21,1 & 20 \\
\hline \multicolumn{3}{|c|}{ Critérios para diagnóstico de dependência } \\
\hline Positivo & 77,9 & 74 \\
\hline Negativo & 22,1 & 21 \\
\hline \multicolumn{3}{|c|}{ Critérios para diagnóstico de abuso } \\
\hline Positivo & 83,1 & 79 \\
\hline Negativo & 16,9 & 16 \\
\hline \multicolumn{3}{|c|}{ Problemas nos últimos 12 meses } \\
\hline Polícia ou justiça & 95,8 & 91 \\
\hline Desemprego & 73,7 & 70 \\
\hline Violência & 51,6 & 49 \\
\hline Luto de amigo & 25,2 & 24 \\
\hline
\end{tabular}

SRQ-20: Self-Reporting Questionnaire.

As diferenças em relação aos estudos de base comunitária sugerem algum tipo de seleção para o acesso de usuários de crack aos serviços do SUS, como parece ocorrer em outras áreas 16,17,18,19,20. As condições clínicas e o padrão de consumo do crack são considerados graves e compatíveis com o descrito em outros estudos $3,16,17$, mas o perfil socioeconômico é diferenciado e isto não se deve à realidade dos municípios, já que, segundo o Instituto Brasileiro de Geografia e Estatística (IBGE; http://www.ibge.gov.br/cidadesat/to pwindow.htm?1, acessado em 11/Jul/2011), no censo demográfico de 2010, a incidência de pobreza era semelhante entre os três municípios, em média: $28,22 \%$. Pessoas com maior comprometimento social parecem não chegar às redes de saúde, o que remete à necessidade dos municípios implementarem estratégias de facilitação do acesso, com maior envolvimento de agentes comunitários de saúde, com os Programas de Redução de Danos (PRD) ou com os Consultórios de Rua, ou outras ações de aproximação entre comunidade e serviços 26,27,28,29. Outra opção é a maior oferta de estratégias focais, centradas na definição de objetivos terapêuticos e na própria motivação para o tratamento 30 .

Nicotina, álcool e maconha foram mencionados como outras drogas consumidas, além do crack, que é usado de modo frequente e pesado, ainda que, de início mais tardio que o descrito em outros estudos no Brasil, que identificaram idades médias de experimentação da droga inferiores aos 18 anos 6,20. A elevada ocorrência de escores positivos para dependência e abuso corroboram os demais trabalhos que avaliaram usuários em atendimento 6,16,20. A ocorrência de problemas com a polícia ou justiça, o desemprego, o envolvimento em situações de violência e luto por amigos, traduzem problemas importantes associados ao uso do crack. A morte de amigos foi a situação de luto mais referida, em índice que parece elevado, considerando-se que se trata de um grupo de adultos jovens. Os escores do SRQ-20 foram compatíveis com os descritos em outros estudos, que descreveram a ocorrência de sintomas depressivos e ansiosos entre usuários de crack e de outras drogas, tanto como consequência do consumo da droga quanto ocorrendo na forma de comorbidade 6,17,18,19,20,31.

A principal limitação do estudo foi reunir um número pequeno de sujeitos. A expectativa inicial do grupo, baseada nas estimativas das equipes dos CAPS, era atingir um total de 200 usuários no período assumido. As razões do reduzido ingresso de pacientes sugeridas pelas equipes nas reuniões de devolução dos dados foram o padrão de registros empregado nos CAPS, a ocorrência de reingressos dos pacientes e as flutuações do mercado do crack. Os registros de casos dos CAPS englobam todos os atendimentos realizados, porém mais da metade dos que envolvem a população usuária de crack não caracteriza acolhimento do usuário no serviço e sim orientação a familiares ou instituições. Em seu papel de referência e regulação nas redes locais, os CAPS têm notícias de usuários em atendimento em outros serviços, cria-se um registro do caso e, pelo menos, a expectativa de futuros atendimentos. 
Tais situações não geraram ingressos no estudo. O reingresso de usuários de crack que iniciam, interrompem e voltam a buscar tratamentos cria uma impressão subjetiva de maior volume e circulação de usuários no serviço, mas não geraram novas entrevistas de ingresso no estudo. $\mathrm{Na}$ percepção das equipes, a busca por atendimento também acompanha oscilações do mercado, que é de um produto ilícito, portanto sensível a movimentos muito peculiares. Isso ocorreu em Igrejinha, onde se detectou um movimento local que estimulava a denúncia à polícia de qualquer pessoa relacionada ao crack, percebido no CAPS como associado à redução da busca por atendimentos no serviço.

O artigo descreve o perfil de 95 usuários de crack acolhidos em três Centros de Atenção Psicossocial (CAPS) da Região Metropolitana de Porto Alegre, no Sul do Brasil, entre agosto de 2009 e março de 2010. Todos os usuários de crack que buscaram atendimento no período foram entrevistados. Utilizou-se questionários desenvolvidos pela equipe, mais o Self-Reporting Questionnaire (SRQ-20) e inventários de critérios de dependência e abuso (SAMHSA). Houve predomínio de pacientes homens, adultos jovens, com escolaridade fundamental, sem ocupação regular, mas com renda individual informada, em uso frequente e pesado há mais de um ano, e a maioria preenchia critérios para dependência e abuso do crack e tinha escores elevados de SRQ-20. Os resultados evidenciam que os CAPS são buscados por usuários de crack em sofrimento, que deve ser valorizado, mas também a existência de algum tipo de seleção na oferta destes serviços, caracterizada pelas especificidades de renda, escolaridade e grupo primário de apoio aos entrevistados.

Cocaína Crack; Usuários de Drogas; Serviços de Saúde Mental

\section{Conclusão}

Recomenda-se, como iniciativa redutora de riscos e de danos, facilitar o acesso de usuários de crack aos serviços do SUS. A seletividade percebida, não descaracteriza a necessidade do atendimento qualificado de quem chega.

Podem ser considerados prioritários para iniciativas de facilitação do acesso de grupos de usuários sem apoio familiar ou vivendo em situação de rua, de baixa ou nenhuma escolaridade e sem renda pessoal ou familiar. Mulheres, especialmente as gestantes, também merecem atenção especial.

Mais estudos e com um número maior de indivíduos são necessários para que se tenha um mapa mais completo das necessidades dessa população.

\section{Colaboradores}

R. L. Horta elaborou o projeto original, coordenou a coleta e análise de dados e a redação da versão final do artigo. B. L. Horta participou da análise de dados e da redação da versão final. A. P. Rosset participou da coleta de dados, realizou a análise e contribuiu na redação da versão final. C. L. Horta fez a revisão da literatura e participou da redação final do artigo. 


\section{Referências}

1. Carlini EA, Galduróz JC, Noto AR, Carlini CM, Oliveira LG, Nappo SA, et al. II levantamento domiciliar sobre o uso de drogas psicotrópicas no Brasil: estudo envolvendo as 108 maiores cidades do Brasil - 2005. São Paulo: Centro Brasileiro de Informações sobre Drogas Psicotrópicas; 2006.

2. Cunha PJ, Nicastri S, Gomes LP, Moino RM, Peluso MA. Alterações neuropsicológicas em dependentes de cocaína/crack internados: dados preliminares. Rev Bras Psiquiatr 2004; 26:103-6.

3. Rodrigues VS, Caminha RM, Horta RL. Déficits cognitivos em pacientes usuários de crack. Rev Bras Ter Cogn 2006; 2:67-72.

4. Kessler F, Pechansky F. Uma visão psiquiátrica sobre o fenômeno do crack na atualidade. Rev Psiquiatr Rio Gd Sul 2008; 30:96-8.

5. Paquette C, Roy É, Petit G, Boivin JF. Predictors of crack cocaine initiation among Montréal street youth: a first look at the phenomenon. Drug Alcohol Depend 2010; 110:85-91.

6. Sanchez ZVDM, Nappo SA. Seqüência de drogas consumidas por usuários de crack e fatores interferentes. Rev Saúde Pública 2002; 36:420-30.

7. Araujo RB, Oliveira MS, Pedroso RS, Miguel AC, Castro MGT. Craving e dependência química: conceito, avaliação e tratamento. J Bras Psiquiatr 2008; 57:57-63.

8. Kasarabada ND, Anglin MD, Khalsa-Denison E, Paredes A. Variations in psychosocial functioning associated with patterns of progression in cocainedependent men. Addict Behav 1998; 23:179-89.

9. Orsi MM, Oliveira MS. Avaliando a motivação para mudança em dependentes de cocaína. Estud Psicol (Campinas) 200; 23:3-12.

10. Sofuoglu M, Brown S, Babb DA, Hatsukami DK. Depressive symptoms modulate the subjective and physiological response to cocaine in humans. Drug Alcohol Depend 2001; 63:131-7.

11. Zaleski M, Laranjeira RR, Marques ACPR, Ratto L, Romano M, Alves HNP. Diretrizes da Associação Brasileira de Estudos do Álcool e Outras Drogas (ABEAD) para o diagnóstico e tratamento de comorbidades psiquiátricas e dependência de álcool e outras substâncias. Rev Bras Psiquiatr 2006; 28:142-8.

12. Ministério da Saúde. Política Nacional de Saúde Mental. http://portal.saude.gov.br/portal/saude/ area.cfm?id_area=925 (acessado em 14/Set/2010).

13. Ministério da Saúde. Abordagens terapêuticas a usuários de cocaína/crack no Sistema Único de Saúde: texto preliminar destinado à consulta pública. http://portal.saude.gov.br/portal/ar quivos/pdf/abordagemsus.pdf (acessado em 10/ Set/2010).

14. Hughes A, Sathe N, Spagnola K. State estimates of substance use from the 2006-2007 National Surveys on Drug Use and Health. Rockville: Office of Applied Studies, Substance Abuse and Mental Health Services Administration; 2009. (NSDUH Series H-35, HHS Publication No. SMA 09-4362).
15. Mari JJ, Iacoponi E, Williams P, Simões O, Silva JBT. Detection of psychiatric morbidity in the primary medical care setting in Brazil. Rev Saúde Pública 1987; 21:501-7.

16. Duailibi LB, Laranjeira R, Ribeiro M. Profile of cocaine and crack users in Brazil. Cad Saúde Pública 2008; 24 Suppl 4:S545-57.

17. Oliveira G, Nappo SA. Caracterização da cultura de crack na cidade de São Paulo: padrão de uso controlado. Rev Saúde Pública 2008; 42:664-71.

18. Fochi EL, Moraes MS, Chiaravalloti FN, Gandolfi D, Ferreira EMA. Caracterização de 46 usuários de crack abordados pelo programa de redução de danos “Tá Limpo". HB Cient 2000; 7:85-91.

19. Ferreira Filho OF, Turchi MD, Laranjeira R, Castelo A. Perfil sociodemográfico e de padrões de uso entre dependentes de cocaína hospitalizados. Rev Saúde Pública 2003; 37:751-9.

20. Guimarães CF, Santos DVV, Freitas RC, Araujo RB. Perfil do usuário de crack e fatores relacionados à criminalidade em unidade de internação para desintoxicação no Hospital Psiquiátrico São Pedro de Porto Alegre (RS). Rev Psiquiatr Rio Gd Sul 2008; 30:101-8.

21. Bungay V, Johnson JL, Varcoe C, Boyd S. Women's health and use of crack cocaine in context: structural and 'everyday' violence. Int J Drug Policy 2010; 21:321-9.

22. Santos AMCC. Articular saúde mental e relações de gênero: dar voz aos sujeitos silenciados. Ciênc Saúde Coletiva 2009; 14:1177-82.

23. Lima MCP, Menezes PR, Carandina L, Cesar CLG, Barros MBA, Goldbaum M. Transtornos mentais comuns e uso de psicofármacos: impacto das condições socioeconômicas. Rev Saúde Pública 2008; 42:717-23.

24. Gama SGN, Szwarcwald CL, Sabroza AR, Castelo BV, Leal MC. Fatores associados à assistência prénatal precária em uma amostra de puérperas adolescentes em maternidades do Município do Rio de Janeiro, 1999-2000. Cad Saúde Pública 2004; 20 Suppl 1:S101-11.

25. Sherman SG, Reuben J, Chapman CS, Lilleston P. Risks associated with crack cocaine smoking among exotic dancers in Baltimore. Drug Alcohol Depend 2011; 114:249-52.

26. Ministério da Saúde. Álcool e drogas: um problema social, e não criminal. http://portal.saude.gov. $\mathrm{br} /$ portal/saude/visualizar_texto.cfm?idtxt $=34647$ (acessado em 14/Nov/2010).

27. Coordenação Geral de Saúde Mental, Álcool e Outras Drogas, Departamento de Ações Programáticas Estratégicas, Secretaria de Atenção à Saúde, Ministério da Saúde. Chamada para seleção de projetos de consultórios de rua e redução de danos. Portaria no. 1.059/GM de 04 de julho de 2005. http://portal.saude.gov.br/portal/arquivos/pdf/ consultorios_de_rua_rd.pdf (acessado em 14/ Nov/2010). 
28. Moraes M. Integral healthcare model for treating problems caused by alcohol and other drugs: perceptions of users, their companions and practitioners. Ciênc Saúde Coletiva 2008; 13:121-33.

29. Faria JG, Schneider DR. O perfil dos usuários do CAPSad-Blumenau e as políticas públicas em saúde mental. Psicol Soc 2009; 21:324-33.

30. Andreta I, Oliveira MS. A técnica da entrevista motivacional na adolescência. Psicol Clín 2005; 17:127-39.
31. Horta LL. Drogas ilícitas - prevalência e fatores associados ao uso em adolescentes de Pelotas - RS [Dissertação de Mestrado]. Pelotas: Universidade Católica de Pelotas; 2002.

Recebido em 03/Abr/2011

Versão final reapresentada em 26/Jul/2011

Aprovado em 22/Ago/2011 